\title{
Undifferentiated Connective Tissue Disease
}

National Cancer Institute

\section{Source}

National Cancer Institute. Undifferentiated Connective Tissue Disease. NCI Thesaurus.

Code C116776.

An autoimmune disorder which does not meet classification criteria used to establish the presence of other well-defined connective tissue diseases. 